\title{
Research Square \\ CONSENSUS: A Shiny application of Dementia evaluation and reporting for the KU ADC Longitudinal Clinical Cohort Database.
}

Palash Sharma ( $\sim$ psharma4@kumc.edu )

University of Kansas Medical Center https://orcid.org/0000-0003-3016-7541

Robert N. Montgomery

University of Kansas Medical Center

Rasinio S. Graves

UC Davis: University of California Davis

Kayla Meyer

University of Kansas Medical Center

Suzanne L Hunt

University of Kansas Medical Center

Eric D. Vidoni

University of Kansas Medical Center

Jonathan D. Mahnken

University of Kansas Medical Center

Russell H. Swerdlow

University of Kansas Medical Center

Jeffrey M. Burns

University of Kansas Medical Center

Dinesh Pal Mudaranthakam

University of Kansas Medical Center

\section{Technical advance}

Keywords: Alzheimer Disease, EDC, REDCap, R-Studio, R-Shiny, Consensus, data management

Posted Date: September 15th, 2020

DOl: https://doi.org/10.21203/rs.3.rs-69396/v1

License: (c) (i) This work is licensed under a Creative Commons Attribution 4.0 International License.

Read Full License 
Version of Record: A version of this preprint was published at JAMIA Open on July 1 st, 2021. See the published version at https://doi.org/10.1093/jamiaopen/ooab060. 


\section{Abstract}

Background: The University of Kansas Alzheimer's Disease Center (KU ADC) maintains several large databases to track participant recruitment, enrollment, and capture various research related activities. It is challenging to manage and coordinate all the research related activities. One of the crucial activities involves capturing the data and maintaining high data quality, which ensures data reusability and reproducibility.

Methods: To effectively manage the cohort, the KU ADC utilizes a combination of open source Electronic Data Capture (EDC) (i.e. REDCap), along with other homegrown data management and visualization systems developed using R-studio and Shiny.

Results: In this manuscript, we describe the method and utility of the user-friendly dashboard that was developed for the rapid reporting of dementia evaluations along with data visualization, which allows clinical researchers to summarize recruitment metrics, automatically generate letters to both participants and health care providers, and depict other key metrics, which ultimately help optimize workflows.

Conclusions: We believe this general framework would be beneficial to any institution for capturing and maintaining similar longitudinal databases for reporting and summarizing key metrics pertaining to their research.

\section{Background}

Alzheimer's disease (AD) is the most common neurodegenerative disorder that leads to a gradual decrease in cognitive abilities [1]. AD contributes to the sixth leading cause of death in the United States and a fifth leading cause in the age group of 65 and over [2]. It is estimated that approximately 5.4 million people have AD in the USA. [2],[4]. Alarmingly, the age-adjusted death rate increased by $39 \%$ from 2000 through 2010 [2]. For these reasons, the National Institutes of Health (NIH), specifically the National Institute on Aging (NIA), fosters AD research by providing federal funding to support Alzheimer's disease Centers (ADCs) at medical institutions throughout the USA [3]. Each ADC and their affiliated researchers work to better understand the underlying risk factors of $A D$ and improve patient care. This is done by conducting translational research and by collecting a substantial, longitudinal, standardized clinical and neuropsychological dataset called the Uniform Data Set (UDS), developed and maintained by the National Alzheimer's Coordinating Center (NACC). As one of 32 ADCs, the KU ADC, through clinical research and critical care, actively works to further the understanding of $A D$ to improve diagnoses, patient care, and educational resources for those affected by AD.

To enhance clinical activities and facilitate decision making strategies, the KU ADC manages many research activities such as study enrollment, patient screening, and research data capture. These activities are exemplified in the KU ADC Clinical Cohort study, which is a prospective, longitudinal study, following around 400 participants, both with and without memory impairment. Each year, participants in the Clinical Cohort complete clinical and neuropsychological evaluations as part of the UDS created by 
NACC. Organizing data capture and monitoring cross-relationships in different research studies is an important function of the Data Management and Statistics (DMS) Core of the KU ADC. Additional uses for these cross-linked data include generation of recruitment metrics for study sponsors and summary communications to participants and their physicians.

Historically, research data has been collected using the paper-based case report forms (CRFs) and was then entered into a database to generate electronic records [5]. Although the data collection process using CRFs is simple, error checking during entry is demanding and involves an additional validation step to ensure data safety and preserve the quality and integrity of the data [5]. In recent years electronic data capture (EDC) has become more prevalent with advances in hardware, software, and internet connectivity. With the broadening use of EDC, administrative bodies in the US and Europe have provided guidelines to assure data safety, privacy, and data interchangeability [6],[7],[8],[9]. Thus, the EDC system provides a platform to gather, manage, and store clinical research data more securely than ever. Electronic data capture allows simple access to the data, with security restrictions in place, which complies with regulatory standards, allows for real-time error checking, and helps maintain data integrity [5],[10]. It is evident through literature that EDC is more time-efficient for data capture and more time saving when completing data validation, while maintaining comparable error rates to paper-based approaches.[5],[11].

The KU ADC uses an open-source EDC system for data capture which has accounted for improved efficiency in the speed of data transfer, consistent and periodic data reporting to the National Alzheimer's Coordinating Center (NACC) [12]. Additional advantages of this system are that it provides reporting in real-time while maintaining data safety. It is prone to fewer transcription errors, and thus overall improved data quality. Our EDC system provides a swift data management flow from initial contact with patients, to final diagnosis stage, to uploading the data to the NACC database, fulfilling the requirements set by NACC [12]. In addition, to further leverage the capabilities of an EDC based system, we have developed and deployed Web applications through which end users can easily find and summarize key variables and metrics to disseminate the clinical outcome and reports for the patients and physicians.

This manuscript aims to describe the process and implementation of a Shiny app to produce real-time reports for weekly diagnosis meetings. This article attempts to summarize our entire consensus process of the KU ADC from data collection to data management and consensus reporting. Specifically, this paper will advocate the idea to use the open-source software and how to automate the workflow to improve efficiency and compliance, and how this process helps facilitate the consensus meeting. Furthermore, we will discuss how the consensus app helps facilitate sending automated correspondences to both participants and their primary care physicians (PCPs) in the form of a thank-you letter to Clinical Cohort participants for their contribution to the KU ADC research and a diagnostic summary letter to PCPs to provide an update on the participant's recent research visit.

\section{Material And Methods \\ Data Collection}


The KU ADC Clinical Cohort is a longitudinal study involving the collection of annual clinical and neuropsychological assessments, for participants with and without dementia [12], [13]. Participants enrolled in the KU ADC Clinical Cohort study receive the required NACC UDS assessments, regulated by NACC guidelines [15], [16], along with several other KU specific items collected for site specific research questions and to aid with operational components of the study. These longitudinal questionnaires and instruments are captured during the participants' initial visit and subsequent follow-up visits, administered by highly trained research personnel, clinicians, and psychometrists. Each participant is accompanied by a family member or close friend as a collateral source for their annual clinical evaluation visit where valuable information about the participant is captured (functional, behavioral, physiological etc.). A research coordinator gathers and updates demographic information, medical history, family history, and medication usage, and administers surveys related to the participants daily functioning and mood. The clinician performs a clinical interview, accompanied with a general neurological exam, to complete a clinical dementia rating scale (CDR) evaluation [13]. Data are directly entered through the REDCap [19] system. A Neuropsychological test battery is also performed by a trained psychometrist following a standardized testing method [12],[13]. These test scores and summary of the neuropsychological battery also follows NACC guidelines to satisfy the standard UDS 3.0 requirements [12],[15]. The number of variables listed for the ADC UDS requirements and the other dataset collection is further illustrated at Katelyn et al. paper [14]. For Consensus reporting, we mostly utilize UDS 3.0 data from the cognitive test and CDR visits. More detailed recruitment methods and protocols were reported under the Graves et al. paper [12]. We recruit our participants through various organizations and identify volunteers across different stages of cognition who wish to contribute to $A D$ research.

\section{Data Management}

Study data were collected and managed using REDCap [19],[20]. REDCap is a secure web-based software platform designed to support data capture for research studies, providing 1) an intuitive interface for validated data capture; 2) audit trails for tracking data manipulation and export procedures; 3 ) automated export procedures for seamless data downloads to common statistical packages; and 4) procedures for data integration and interoperability with external sources [19],[20]. A variety of open-source software is used to create the Shiny Dashboard that is utilized during the weekly consensus meetings. Data cleaning and visualization are done through $\mathrm{R}, \mathrm{R}$ studio, $\mathrm{R}$ markdown, and Shiny. $\mathrm{R}$ is an open-source programming language software for statistical computing and supported by R Foundation for Statistical Computing [21]. $\mathrm{R}$ is freely available under the GNU General Public License. R has versatile flexibility including custom user packages, graphical devices, import/export capabilities, and reporting (Knitr [22], Sweave) etc. Primarily, we use R studio for data transformation, report generation and data visualization. R Studio [25] is also an open-source IDE (Integrated Development Environment) software for R. R studio is available as an open-source and commercial edition and can be deployed in any operating. The advantage of using R Studio is that it can run on a server that enables multiple users to access the tools simultaneously and when paired with the Shiny package can seamlessly develop a web-based dashboard. HTML (Hypertext Markup Language) reports used in consensus diagnosis meetings are 
generated by the R Markdown package from $\mathrm{R}$ library [23]. Shiny is an $\mathrm{R}$ package used to build an interactive Web application and dashboard [24]. We use the Shiny package which is hosted on a dedicated server to build and host real-time data visualization and consensus reporting for our longitudinal clinical cohort study data. Utilizing open-source software has gained more fame in the research group due to the cost benefits, availability, and capabilities of working functionality across platforms.

\section{Data Security}

A primary concern using open source software such as R, Python etc., is to make sure the data privacy and vulnerability are in place, similar to that of a closed system [17]. The balance between ease of access for relevant researchers with protecting a patient's data must always lean in favor of the security of the data. The free version of Shiny does not provide a way to secure access to apps through a login screen, and while there are templates for $\mathrm{R}$ code that will do this, the security that these login screens provide is questionable. To deal with this challenge, our Shiny app is integrated with KUMC's Central Authentication Service (CAS) system. It grants a group of users' access the shiny app with the single sign-on credential facilitated by the University of Kansas medical center (KUMC). Similarly, REDCap is also linked to the KUMC CAS system to serve an extra layer of protection. All programs written by the programmers, coding of variables, outputs are stored in the secured server hosted by the Data Core Management and Statistics Core of the KUADC. External users or collaborators can submit a written request for permission to access the shared drive, shiny App server or any REDCap project associated with the consensus cohort database.

Figure 1: An overview of the Workflow of the consensus Reporting using R shiny App

\section{Process}

The KU ADC employs multiple projects in REDCap to capture various phases of participation. Initial research participation inquiries and study participation records are captured in our Participant Relations and Outreach Management Tool (PROMPT database) [18]. Clinical Cohort participant evaluations and tracking are performed in a separate database. A third database is used to track all past and ongoing human subject's research studies. Upon compilation, all data is corroborated into a single REDCap project. A sketch of the process and workflow is displayed in Fig. 1. Data exports to the secure server are automatic and implemented via a task schedular aka "cron job". The cron job executes a series of python code, which pulls down the exports listed in the bootstrap project. The bootstrap project/process is a REDCap project that lists all the relevant information (API key, fields to exports, and project information) so that the python code can create the exports. Then the python code creates requests for each export listed in the bootstrap project and calls for the REDCap API. Next, the code generates the CSV files with each export's returns results. Finally, a shell script moves the generated CSV files securely to the desired server location. Once all the data dumps are completed, a series of $\mathrm{R}$ scripts is utilized to join all the datasets that come from various projects and creates a final dataset that is later loaded into a shiny app for Consensus reporting and other purposes. The front-end shiny App user is not required to know any programming language. They can generate a participant's consensus report (usually in the Markdown 
format/ HTML format) by browsing and navigating the Shiny dashboard tab. Once the appropriate PTID (Patient's unique identifier) is selected, code will run on the backend and display the reports within a second. We incorporated multiple features into our shiny App based on the need for the project and reporting metrics. Figure 2,3 provides an overview of the Shiny App dashboard.

Figure 2: An overview of the Consensus App utilizing Shiny Dashboard

Figure 3: PCP letter creating Tab utilizing Shiny Dashboard

\section{Results}

In this section, we illustrate various advanced functioning of this analytic dashboard. We previously reviewed [12] the efficiency of electronic vs. paper source, time to consensus diagnosis, and completion. However, this paper emphasized some detailed features by leveraging the computational power from the app and how this interface serves to promote the physician-patient experience to ultimately contribute to further decision making for AD diagnoses.

The consensus app is run twice a week the 5 to 10 participants who on average evaluated each week. It vastly reduced the time to generate letters for individual persons periodically. Before adaptation of shiny app, the median number of hours required to generate letters for each participant is 1 hour, where we can now generate letters within 13-15 minute. Additionally, it allows the administrator to go back and refer letters which were generated in the past. Also, this EDC based system improved time to generate consensus reports for weekly meeting. Overall, we can say that, after implementation of the consensus app, we can significantly reduce the time and cost for a large longitudinal cohort with an extended followup period. Next, we will discuss how this app helps to send automated preformatted letters to both participants and their primary care physicians (PCPs) to Clinical Cohort participants. A flowchart of the inperson and telephone visit information is shown in Fig. 4.

\section{Consensus Report}

Consensus reports are the standard reports that are generated for the consensus meetings. This iteration of consensus has been designed to reduce user interaction error as much as possible. Through this interface the user is not allowed to input the incorrect combination of the patient's unique ID number, visit year, etc., and it produces the standard reports along with the cognitive scores for each participant visit. Furthermore, it also displays the information of the KU ADC clinician, psychometrist, and intake coordinator who administered the CDR, neuropsychological cognitive test, and collected demographic information, respectively. Every week a group of KU ADC physicians/practitioners/neurologists/psychometrist join the consensus meeting along with other study staff to review participant cases. The decision of a patient's diagnosis is based on the results of cognitive testing, study partner comments, and the general impression and observations of the clinician who saw the participant. All relevant and necessary information is compiled into the consensus reports for ease of access and simplicity, making reviewing each case more streamlined. In addition, this app can also 
generate consensus reports for the participants who have reached the stages of dementia where they cannot continue in-person study visits and instead are followed through telephone contact and evaluations. However, in this case, there will be no cognitive scores available and the reports may or may not have a participant rational, depending on the functional ability of the participant.

\section{Primary Care Physician (PCP) letter}

Figures 2 and 3 display that the CONSENSUS app can generate both the regular, in-person and telephone follow-up versions of the PCP letter. This letter includes the physician's name, street address, city, state, zip code, and final impression from the attending ADC clinician or the impression from the telephone follow-up interview. The dataset used to create PCP letters must be present for each of their respective variable names. This app does not generate PCP letters if the participant did not opt in to have their clinical evaluation summary shared with their doctor. In REDCap, the study coordinator selects a variable indicating either "yes" the participant has consented to the PCP letter or "no", the participant has elected not to have a PCP letter sent. Based upon this variable, the CONSENSUS app determines whether a PCP letter can be created and looks for the presence of additional variables needed to complete the request, namely physician name and address. The same mechanism applies for telephone follow up PCP letters. If the general structure of the variable name is validated, selecting an available participant's ID will produce the physician's address along with the final impression from the attending physician or interviewer. The app pulls this information into a standardized letter template and saves a separate file with the PCP address, making printing both the letter and mailing envelopes simpler.

\section{Participant Thank you letter}

Like the PCP letter, this Shiny app can be used to send thank you letters to the participants. If the participant's street address, city, state, and zip code are not filled out correctly, the end-user will not be given the options to generate a letter. Additionally, under this tab, it allows to select whether the participants are part of the KU ADC single evaluation Registry Study, or if part of the longitudinal Clinical Cohort. Depending on the selection, the app will select the appropriate letter template, ensuring the correct language is used, minimizing end user confusion. Finally, this function will produce a pre-formatted Thank You letter to the participants which includes individualized feedback to the participant. We add a flow diagram (Fig. 4) to help visualize how in-person and telephone follow up visits work for different functionality.

\section{Scheduling letter}

This Shiny interface also can generate scheduling reminder letters for the participants with expected longitudinal follow-up in the KU ADC Clinical Cohort. As shown in Figs. 2 and 3, selecting a month from the "Scheduling letters" tab will reveal the number of 'regular' in-person Cohort and Telephone follow-up anniversaries are expected for that month. Running the "Generate letters" tab from the subsection of the "Scheduling letters" tab will produce letters for all the listed participants. However, if someone had the following criteria such as screen failures, initial evaluations only, discontinued, deceased, minimal 
contact, or Registry only, this app will not produce a scheduling letter for those participants, as continued follow-up is not expected.

Figure 4: Flow diagram of in-person and Follow up visit for different functionality.

\section{Discussion}

In clinical and translation research, designing and implementing dashboard-based data integration and analytic visualization has gained popularity. [26],[27],[28],[29],[30]. Application-based infrastructure helps clinicians to better inform the patient's behavior and medical history and guide them to make the best decision of the diagnosis [26], [27]. To the best of our knowledge, the consensus app is one of the first open source-based application systems designed for rapid reporting of dementia evaluation and visualization. Exploiting the modern computational and programming power, this framework is adaptable for other clinical research-based reporting systems.

In this paper, we have discussed the advantages of EDC for longitudinal data in a clinical trial setting. Furthermore, we have implemented an R-shiny based open-source clinical dashboard reporting framework for generating real-time consensus reporting and generating PCP, thank you, and scheduling letters for study participants. This CONSENSUS app uses REDCap for data collection, R packages for data management, standardization of the data, and to generate consensus reports. It also helps display a consensus report along with a quick calculation of recruitment metrics, automatic dissemination of letters, and cognitive scores.

This interactive web application has some attractive features to aid physicians and researchers in getting better insight into the progression of $A D$ of the individual patient and helps inform pertinent longitudinal and follow-up information. Also, this app helps the physicians improve screening time for trials. Participants can also benefit from this process by receiving their feedback quicker through the "thank you" letter. It has an intuitive graphical user interface (GUI). It is accessible via the internet within the KUMC network and can be also used through the web browser without the need for installation. This allows physicians and clinicians to easily navigate through the entire visit with a participant in our research clinic, retrieving required information and data quickly through this program, instead of pulling the records from the volume of paper files.

Adaptation of an open source-based framework has limitations too. There is always a concern utilizing such user-friendly web-based apps for clinical data evaluation and implementation over commercial counterparts. Furthermore, individuals need to have appropriate experience to use technologies and need proper training and logistics to enter data. Another concern is the privacy and security of data management over the internet and for that reason, technical support needs more than the community support. Finally, it requires time and extensive programming skills to develop such sophisticated systems, and maintenance is sometimes costly. Open-source software packages always update their system/package without any warranty, which might lead to the unexpected errors if not updated regularly. 


\section{Conclusions}

In conclusion, the CONSENSUS app plays a vital role in KU ADC Alzheimer's disease research. It is expected that sending preformatted letters of appreciation, scheduling reminders, and minimal visit feedback to participants helps to acknowledge the patient's role in our KU ADC cohort, which ultimately will help with participant retention. Another expected benefit of this application is that by automating physician notification letters, the participant's primary care doctor will receive feedback from highly skilled and trained dementia experts faster, thus the quality of the physician-patient experience will improve. We believe that the adaptation of such an open-source system can not only minimize healthcare costs but also increase the efficiency of the overall healthcare assessment. We envision that the implementation of the consensus-based report system, in a broader spectrum, will enhance the prognosis of dementia-related neurodegenerative diseases and improve patient outcomes.

\section{Declarations}

\section{Ethics approval and consent to participate}

The University of Kansas Medical center approved the study. Informed consent was obtained from each study participant.

\section{Funding}

This research was supported by NIH grant P30 AG035982 through the National Institute on Aging.

\section{Data Sharing Statement}

Code and data related to Shiny App is the proprietary property of the KU ADC. Data sharing is available upon reasonable request pending upon the approvals of IRB and HIPPA consent.

\section{Supplementary Materials}

None

\section{Conflict of Interest}

None to report

\section{Author Contribution}

PS, NM, DPM, KM contributed to the overall planning and writing of the manuscript. RG, EV, NM, PS contributes to the development of the App. All authors have contributed to the writing and review of the final manuscript. All authors approved their contributions and the final draft of the manuscript.

\section{Acknowledgements}




\section{Corresponding Author}

Correspondence to Dinesh Pal Mudaranthakam at dmudaranthakam@kumc.edu

\section{References}

1. Selkoe DJ, Lansbury PJ Jr. Alzheimer's disease is the most common neurodegenerative disorder. Basic Neurochemistry: molecular, cellular and medical aspects. 1999;6:101-2.

2. Tejada-Vera B. Mortality from Alzheimer's disease in the United States: data for 2000 and 2010. US Department of Health and Human Services, Centers for Disease Control and Prevention, National Center for Health Statistics; 2013.

3. National Institute on Aging. Alzheimer's disease Research Centers.

4. Available. https://www.nia.nih.gov/health/alzheimers-disease-research-centers.

5. Alzheimer's Association. Alzheimer's disease facts and figures, Alzheimer's \& dementia Avaiable: https://www.alz.org/alzheimers-dementia/facts-figures.

6. Walther B, Hossin S, Townend J, Abernethy N, Parker D, Jeffries D. Comparison of electronic data capture (EDC) with the standard data capture method for clinical trial data. PloS one. 2011 Sep 23;6(9):e25348.

7. U.S. Department of Health \& Human Services. The Health Insurance Portability and Accountability Act (HIPPA) Privacy and Security Rules. 1996. Available: https://www.hhs.gov/hipaa/forprofessionals/security/laws-regulations/index.html.

8. U.S. Department of Health \& Human Services. The Patient Safety and Quality Improvement Act (PSQIA). 2005. Available: https://www.hhs.gov/hipaa/for-professionals/patient-safety/statute-andrule/index.html.

9. European Parliament. Directive 2002/58/EC of the European Parliament and of the Council of 12 July 2002 concerning the processing of personal data and the protection of privacy in the electronic communications sector (Directive on privacy and electronic communications). 2002. Available: http://eurlex.europa.eu/LexUriServ/LexUriServ.do?uri=CELEX:32002L0058:EN:NOT.

10. U.S. Department of Health and Human Services (FDA) Guidance for Industry - Computerized Systems Used in Clinical Investigations. 2007. Available: http://www.fda.gov/OHRMS/DOCKETS/98fr/04d0440-gdl0002.PDF.

11. Shah J, Rajgor D, Pradhan S, McCready M, Zaveri A, Pietrobon R. Electronic data capture for registries and clinical trials in orthopaedic surgery: open source versus commercial systems. Clinical Orthopaedics and Related Research ${ }^{\circledR} .2010$ Oct 1;468(10):2664-71.

12. Bart T. Comparison of electronic data capture with paper data collection-is there really an advantage. Business Briefing Pharmatech. 2003;Mar:1-4. 
13. Graves RS, Mahnken JD, Swerdlow RH, Burns JM, Price C, Amstein B, Hunt SL, Brown L, Adagarla B, Vidoni ED. Open-source, rapid reporting of dementia evaluations. Journal of registry management. 2015;42(3):111.

14. Swerdlow RH, Hui D, Chalise P, Sharma P, Wang X, Andrews SJ, Pa J, Mahnken JD, Morris J, Wilkins HM, Burns JM. Exploratory analysis of mtDNA haplogroups in two Alzheimer's longitudinal cohorts. Alzheimer's \& Dementia. 2020 Jun 16.

15. McKenzie KA, Hunt SL, Hulshof G, Mudaranthakam DP, Meyer K, Vidoni ED, Burns JM, Mahnken JD. A semi-automated pipeline for fulfillment of resource requests from a longitudinal Alzheimer's disease registry. JAMIA open. 2019 Dec;2(4):516-20.

16. National Alzheimer's Coordinating Center. Uniform Data Set (UDS). 2015. Available: https://www.alz.washington.edu/WEB/forms_uds.html.

17. National Institute on Aging. National Alzheimer's Coordinating Center (NACC). 1999. Available: https://www.nia.nih.gov/research/dn/national-alzheimers-coordinating-center-nacc.

18. Kruse CS, Smith B, Vanderlinden H, Nealand A. Security techniques for the electronic health records. Journal of medical systems. 2017 Aug 1;41(8):127.

19. Vidoni ED, Bothwell RJ, Dwyer JR, Burns JM. Novel recruitment models will drive Alzheimer's trial success. Alzheimer's \& dementia: the journal of the Alzheimer's Association. 2018 Jan;14(1):117.

20. Harris PA, Taylor R, Thielke R, Payne J, Gonzalez N, Conde JG. A metadata-driven methodology and workflow process for providing translational research informatics support. J Biomed Inform. 2009;42(2):377-81.

21. Harris PA, Taylor R, Minor BL, Elliott V, Fernandez M, O'Neal L, McLeod L, Delacqua G, Delacqua F, Kirby J, Duda SN. The REDCap consortium: Building an international community of software platform partners. Journal of biomedical informatics. 2019 Jul 1;95:103208.

22. R: A Language and Environment for Statistical Computing [computer program] R Foundation for Statistical Computing; Vienna, Austria: 2013. URL: http://www.r-project.org.

23. knitr[computer program] 2014 URL: Available: http://rmarkdown.rstudio.com.

24. R Markdown [computer program] 2015 Version 2. Available: http://yihui.name/knitr/.

25. Studio R. Inc. shiny: Web Application Framework for R. R package version 0.10. 0. 2014.

26. Allaire J.. Boston. RStudio: integrated development environment for R. MA. 2012;770:394.

27. Dagliati A, Sacchi L, Tibollo V, Cogni G, Teliti M, Martinez-Millana A, Traver V, Segagni D, Posada J, Ottaviano M, Fico G. A dashboard-based system for supporting diabetes care. J Am Med Inform Assoc. 2018 May;25(5):538-47.

28. Tenenbaum JD, Avillach P, Benham-Hutchins M, Breitenstein MK, Crowgey EL, Hoffman MA, Jiang X, Madhavan S, Mattison JE, Nagarajan R, Ray B. An informatics research agenda to support precision medicine: seven key areas. Journal of the American Medical Informatics Association. 2016 Jul 1;23(4):791-5. 
29. Badgeley MA, Shameer K, Glicksberg BS, Tomlinson MS, Levin MA, McCormick PJ, Kasarskis A, Reich DL, Dudley JT. EHDViz: clinical dashboard development using open-source technologies. BMJ open. 2016 Mar 1;6(3).

30. Khairat SS, Dukkipati A, Lauria HA, Bice T, Travers D, Carson SS. The impact of visualization dashboards on quality of care and clinician satisfaction: integrative literature review. JMIR human factors. 2018;5(2):e22.

31. Wahi MM, Dukach N. Visualizing infection surveillance data for policymaking using Open Source Dashboarding. Applied clinical informatics. 2019 May;10(03):534-42.

\section{Figures}

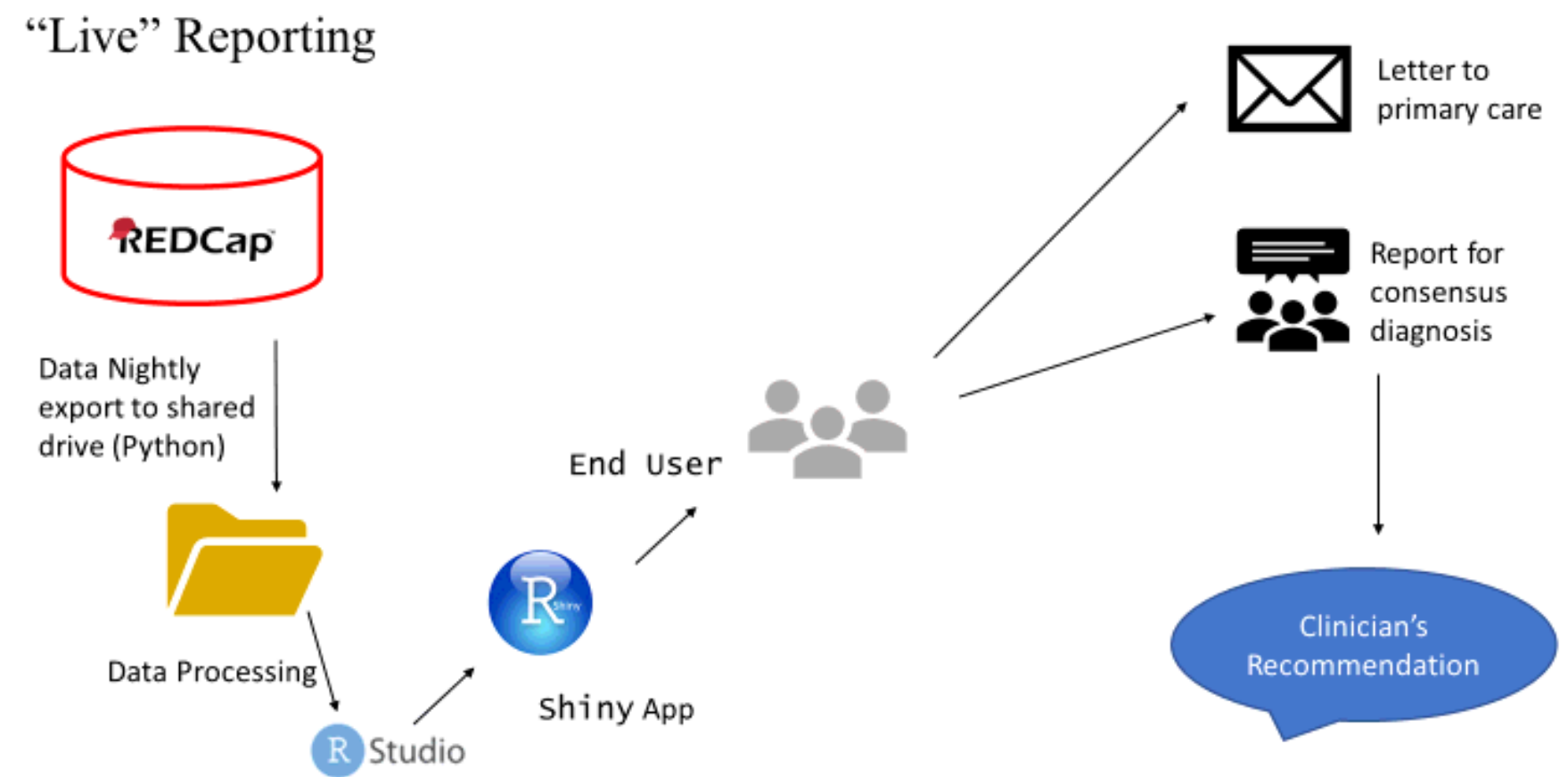

\section{Figure 1}

An overview of the Workflow of the consensus Reporting using R shiny App 


\section{KU ADC Consensus Reporting Tool $\equiv$}

Consensus Reports

Consensus Reports (Telephone)

$\triangle$ PCP Letters

D PCP Letters (Telephone)

$\triangle$ Participant Thank You Letters

iㅛ Scheduling Letters

i Information

Exit
Consensus Report (Telephone Followups)

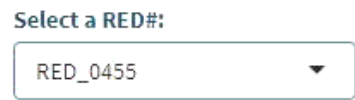

Select a Visit Year:

$2015 \quad-$

Select a Visit Number:

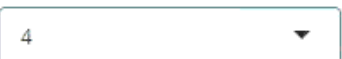

Run Consensus Report (Tele)

\section{ADC Staff Associations}

If you get conflicting information regarding visit number and visit year, the report will not run and will crash. Example: if you see participant RED_0000 has T4 for 2016 on the folder but when you pull up RED_CO00 here (to the left) they have visit number 5 associated with year 2016, DO NOT run this participant. Click 'Generate ADC Staff' to reveal those responsible and go on to the next participant.

Attending Physician:

Intake Coordinator Initials:

Clear ADC Staff Info

Update ADC Staff Info

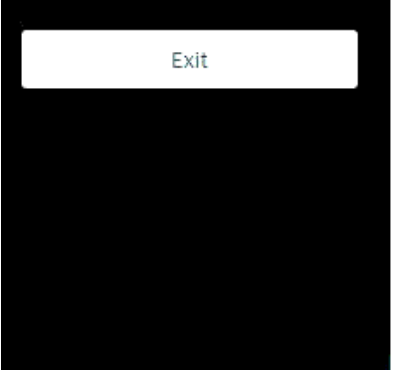

Figure 2

An overview of the Consensus App utilizing Shiny Dashboard

\section{KU ADC Consensus Reporting Tool $\equiv$}

E Consensus Reports

L Consensus Reports (Telephone)

$\square$ PCP Letters

D PCP Letters (Telephone)

Participant Thank You Letters

\# Scheduling Letters

i. Information

Exit

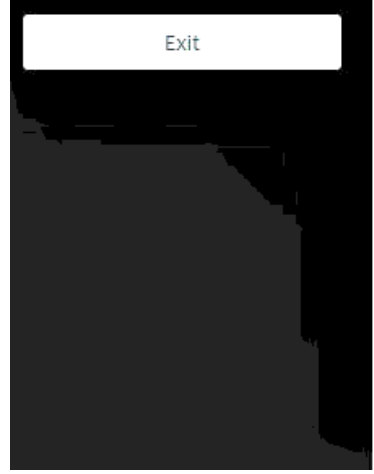

Primary Care Physician Letter

Select a RED\#:

RED_0179 $\quad \longrightarrow$

Select a Visit Year:

$2012 \quad \longrightarrow$

Select a Visit Number:

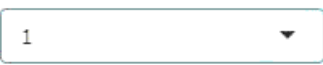

Does the participant want a letter sent to their physician?

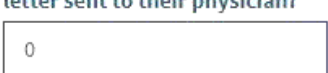

$1=$ Yes; $0=$ No

No PCP letter can be produced.
Physician Information

*Physician Name:

*Street Address:

*City:

* State:

*Zipcode:

ADC Staff Associations

* - Each field above must be filled out. Click the 'Update ADC Staff' if Physician's information is incomplete and the participant wants to send a letter.

Attending Physician:

*Final Impression from Attending Physician:

Cog Tester:

Intake Coordinator Initials:

Clear ADC Staff Info

Figure 3

PCP letter creating Tab utilizing Shiny Dashboard 


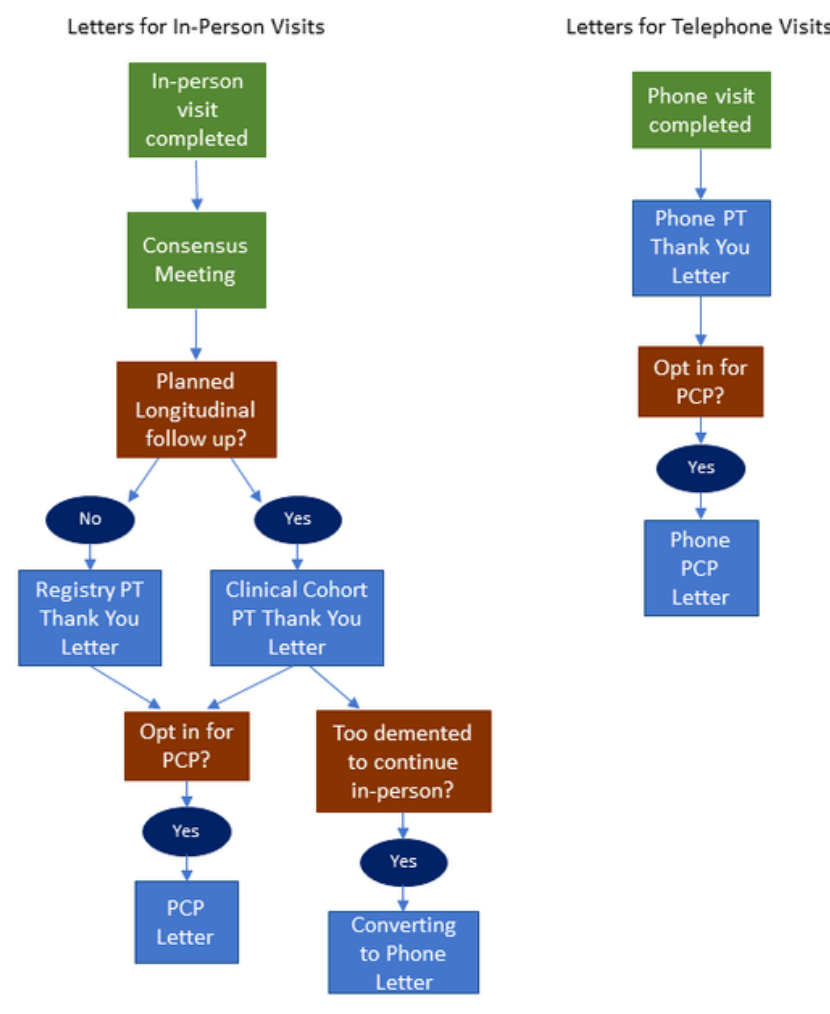

Figure 4

Flow diagram of in-person and Follow up visit for different functionality. 